\title{
Cardiac sarcoidosis: a comprehensive review
}

\author{
Vishal Sekhri' ${ }^{1}$, Shireen Sanal', Lawrence J. DeLorenzo' ${ }^{1}$, Wilbert S. Aronow ${ }^{1,2}$, George P. Maguire
}

1Division of Pulmonary, Critical Care and Sleep Medicine, New York Medical College, Valhalla, NY, USA

2Division of Cardiology, New York Medical College, Valhalla, NY, USA

Submitted: 9 November 2010

Accepted: 31 January 2011

Arch Med Sci 2011; 7, 4: 546-554

DOI: 10.5114 /aoms.2011.24118

Copyright $\odot 2011$ Termedia \& Banach
Corresponding author: Wilbert S. Aronow MD, FACC, FAHA, FCCP

Cardiology Division New York Medical College Macy Pavilion, Room 138 Valhalla, NY 10595, USA Phone: (914) 493-5311 Fax: (914)-235-6274 E-mail:wsaronow@aol.com

\begin{abstract}
Sarcoidosis is a multisystem granulomatous disease of unknown etiology characterized by noncaseating granulomas in involved organs. Organs involved with sarcoidosis include lymph nodes, skin, lung, central nervous system, and eye. Only $40-50 \%$ of patients with cardiac sarcoidosis diagnosed at autopsy have the diagnosis made during their lifetime. Cardiac sarcoidosis can manifest itself as complete heart block, ventricular arrhythmias, congestive heart failure, pericardial effusion, pulmonary hypertension, and ventricular aneurysms. Diagnostic tests such as the electrocardiogram, two-dimensional echocardiography, cardiac magnetic resonance imaging, positron emission tomography scan, radionuclide scan, and endomyocardial biopsy can be helpful in the early detection of cardiac sarcoidosis. Considering the increased risk of sudden death, cardiac sarcoidosis is an indication for early treatment with corticosteroids or other immunosuppressive agents. Other treatments include placement of a pacemaker or implantable defibrillator to prevent sudden death. In refractory cases, cardiac transplantation should be considered.
\end{abstract}

Key words: sarcoidosis, noncaseating granulomas, cardiac sarcoidosis.

\section{Introduction}

Sarcoidosis is a multisystem granulomatous disease of unknown etiology characterized by the presence of noncaseating granulomas in the involved organs. The prevalence is $10-40 / 100,000$ persons in the United States and Europe with an increased prevalence of sarcoidosis in AfricanAmericans compared to Caucasians with a ratio ranging from 10-17 : 1 [1]. The Scandinavian population has a higher prevalence of sarcoidosis than other whites [2].

Sarcoidosis is more prevalent in women than in men. Virtually any body tissue may be involved. Organs commonly involved with sarcoidosis include lymph nodes, skin, lung, central nervous system, and eye. Although many patients may not manifest symptoms at the time of diagnosis, some patients present with systemic symptoms such as fatigue, anorexia, weight loss, and fever. Many patients report dyspnea on exertion, retrosternal chest pain, and cough. In $20 \%$ to $50 \%$ of patients with more acute presentations, the constellation of erythema nodosum, bilateral hilar lymphadenopathy, and polyarthralgia (Lofgren's syndrome) is seen. In the United States, more than half of patients present with chronic respiratory symptoms and few constitutional symptoms [2]. 
Cardiac involvement in sarcoidosis occurs in 20$30 \%$ of patients in pathology series [3]. Cardiac involvement in patients with sarcoidosis is being increasingly recognized and is associated with a poor prognosis. Silverman et al. reviewed 84 autopsy cases of pulmonary sarcoidosis and found myocardial granulomas in $27 \%$ of patients [4]. Cardiac involvement may be high as $58 \%$ in Japanese patients with sarcoidosis $[5,6]$ and may be responsible for as many as $85 \%$ of deaths of Japanese patients with sarcoidosis [6, 7]. Despite these findings, only $5 \%$ of patients with sarcoidosis have clinical manifestations of cardiac disease, and only $40-50 \%$ of patients with cardiac sarcoidosis at autopsy have the correct diagnosis made during their lifetime.

The etiology of cardiac sarcoidosis remains unknown. Environmental, occupational, and infectious causes have been hypothesized. These agents may act as immunologic triggers in genetically predisposed individuals. A multicenter case controlled study did not discover a single predominant cause of sarcoidosis, but identified several exposures that were linked to sarcoidosis risk including agricultural employment and exposure to insecticides or microbial bioaerosols [8]. A number of infectious organisms, including Mycobacteria, Propionibacteria, Borrelia, Rickettsia, and Herpes virus have been implicated as possible etiologies of sarcoidosis [9]. A provocative pilot study reported the empiric use of anti-fungal agents with corticosteroids for 3-6 months in 18 patients resulted in improvement of clinical symptoms, chest $X$-ray infiltration, and pulmonary function [10]. The role of infectious organisms in the etiology of sarcoidosis remains to be clarified. Myocardial sarcoidosis affects young and middle-aged patients without sex predilection. Myocardial involvement may occur in $25 \%$ of patients with sarcoidosis in the United States, and may account for as many as $13-25 \%$ of deaths from sarcoidosis.

\section{Pathology}

The etiology of sarcoidosis is unknown, but the principal inciting event leads to granuloma formation, which can then either resolve or progress to fibrosis. Sarcoidosis requires at least 3 major events: exposure to antigen; acquired cellular immunity directed against the antigen mediated through antigen presenting cells and antigen specific T lymphocytes; and the appearance of immune effector cells that promote a more nonspecific inflammatory response.

The characteristic lesion of sarcoidosis is a discrete, compact, noncaseating epitheloid cell granuloma. The granuloma consists of highly differentiated mononuclear phagocytes and lymphocytes. Infectious and environmental agents have been implicated as potential antigens. These antigens are thought to trigger primarily the helper inducer $T$ cells leading to formation of granuloma lesions. Early in the disease, sarcoid infiltrate mainly consist of mononuclear phagocytes and CD4 positive T cells with a T helper Type 1 response, secreting interleukin-2 and interferon- $\gamma$. At a later stage of lesion evolution, there is a shift of cytokine profile to that of T helper type 2 response which has been demonstrated during the fibroblastic phase of the granuloma and is believed to exert anti-inflammatory effects and result in tissue scarring (Figure 1). Furthermore, high concentrations of interleukin- 6 are found in the circulation at the onset of disease and before the initiation of immunosuppressive therapy, but not thereafter. Interleukin-6 is thought to be involved in the maintenance of inflammation by inducing the proliferation of T cells [11].

Clinical manifestations of sarcoidosis are dependent on both the profusion and location of granulomas. Cardiac sarcoidosis is associated with noncaseating granulomas which may involve the left ventricular free wall, basal ventricular septum, right ventricle, papillary muscles, right atrium, and left atrium [12]. The pathologic features include 3 successive histological stages: edema, granulomatous infiltration, and fibrosis leading to postinflammatory scarring. Pathologic samples of

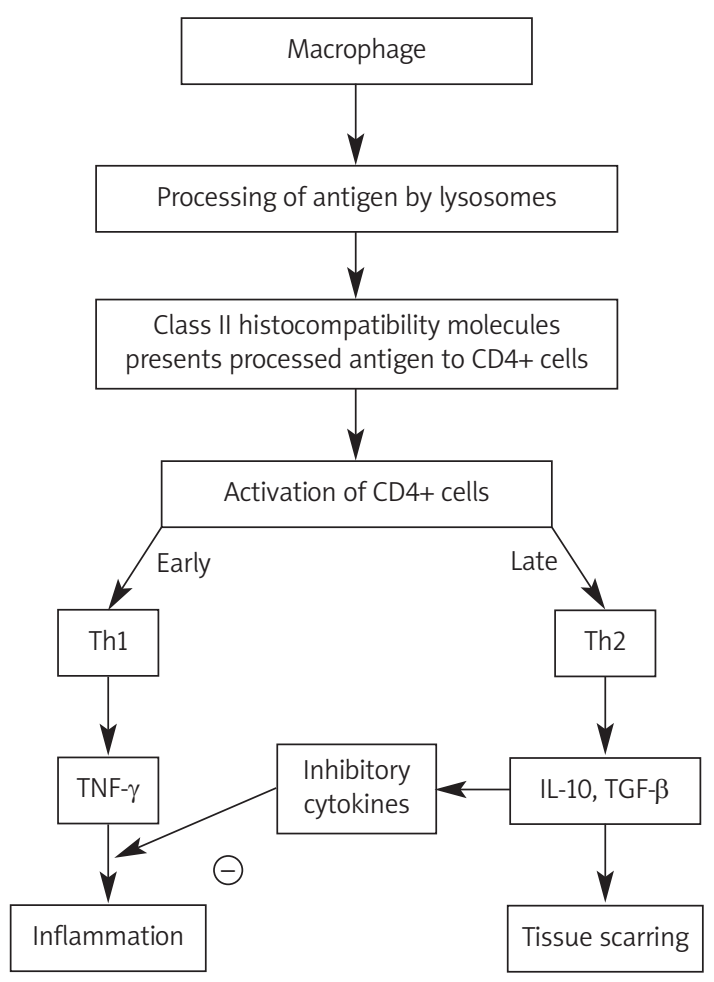

Figure 1. This figure illustrates the pathogenesis of cardiac sarcoidosis 
Table I. Prevalence of cardiac findings in cardiac sarcoidosis

\begin{tabular}{|lc|}
\hline Complete heart block & $23-30 \%$ \\
\hline Bundle branch block & $12-32 \%$ \\
\hline Ventricular tachycardia & $23 \%$ \\
\hline Congestive heart failure & $25-75 \%$ \\
\hline Sudden death & $25-65 \%$ \\
\hline
\end{tabular}

myocardium involved with sarcoidosis reveal the presence of numerous lymphocytes located at the border zones around the granulomas. A dense band of fibroblasts, collagen fibers, and proteoglycans usually encase this aggregate of inflammatory cells [12].

\section{Genetic factors}

A Case Controlled Etiologic Sarcoidosis Study (ACCESS) concluded that first-degree relatives of patients with sarcoidosis had a relative risk of sarcoidosis that was five times that of control subjects [13]. Genetic factors appear to play a role in defining the risk of the disease. On exposure to antigens, some individuals trigger an exaggerated cellular immune response and the formation of granulomas which may be due to genetic predisposition. The genetic factors also determine the pattern of disease, its severity, and prognosis. Monozygotic twins are more commonly affected than dizygotic twins. Familial clusters occur with a rate of at least 19\% in affected black families and $5 \%$ in white families. Multiple serologic studies have identified primary associations with class 1 HLA-A1 and B8 and class II HLA-DR3 in whites [2]. The occurrence of cardiac sarcoidosis in Japanese female patients is associated with the presence of HLA-DQB1*0601 and the allele TNFA2 [14, 15].

\section{Clinical manifestations}

Sarcoidosis can be widespread, or limited to involvement of only a single system at a time. Many asymptomatic cases may be discovered by chest radiography which may or may not progress to clinically symptomatic disease. However, a proportion of patients may present with systemic symptoms which include fever, fatigue, malaise, and weight loss. Intrathoracic involvement occurs in more than $90 \%$ of patients. About one-third of patients have palpable peripheral lymph nodes. Abnormalities of liver function tests are common. Cutaneous involvement may occur in $25 \%$ of patients and may present as erythema nodosum, granulomatous nodule, or papules. Other organ systems may be involved including eye, nervous system, gastrointestinal tract, hematological, parotid, endocrine, reproductive organs, and kidneys.
Cardiac involvement occurs in $20-27 \%$ of sarcoid patients in the United States and may be as high as $58 \%$ in Japan [6]. If cardiac manifestations occur in a patient with multi-systemic sarcoidosis, the diagnosis, although circumstantial, is strongly suspected. However, when cardiac dysfunction is the only manifestation of sarcoidosis, the diagnosis is frequently not entertained [16]. The patients who come with only the symptoms in Table I and are suspected to have cardiac sarcoidosis should undergo a detailed investigation including myocardial biopsy if needed. Even when the diagnosis is considered, it is not often confirmed because of unavailability of specific diagnostic tests.

Early diagnosis and treatment is essential since treatment improves prognosis. The failure to diagnose cardiac sarcoidosis is partly attributable to the relative rarity of clinically apparent forms of the disease. Only $40-50 \%$ of patients with cardiac sarcoidosis at necropsy have clinical evidence of myocardial involvement during lifetime [4]. Serious cardiac dysfunction is detected in $5 \%$ to $10 \%$ of cases. In a significant proportion of patients with cardiac sarcoidosis, the initial presentation is sudden death. In $37 \%$ of patients with cardiac involvement, there were no clinical signs or symptoms of the disease [4].

\section{Complete heart block}

Complete heart block is one of the most common finding in patients with clinically evident cardiac sarcoidosis and occurs at a younger age in patients with sarcoidosis than in patients with complete heart block of other causes [17]. Complete heart block (Figure 2) and bundle branch block have been reported in $23-30 \%$ and $12-32 \%$, respectively of patients with myocardial sarcoidosis. They are caused by involvement of the basal septum by scar tissue, granulomas, or involvement of the nodal artery causing ischemia in the conduction system [6]. Sudden death may be a direct result of complete heart block. For Japanese patients, notably women over 50 years of age, complete heart block is frequent and leads to the diagnosis of cardiac sarcoidosis in $11 \%$ of cases [18]. Patients with sarcoidosis who present with syncope or presyncope should be evaluated for complete heart block with electrocardiographic and Holter monitoring. Sarcoidosis should be included in the evaluation of patients with syncope, especially when it occurs in a younger patient.

\section{Ventricular arrhythmias}

Sudden death caused by ventricular tachyarrhythmias or complete heart block may account for $25-65 \%$ of deaths caused by cardiac sarcoidosis, 


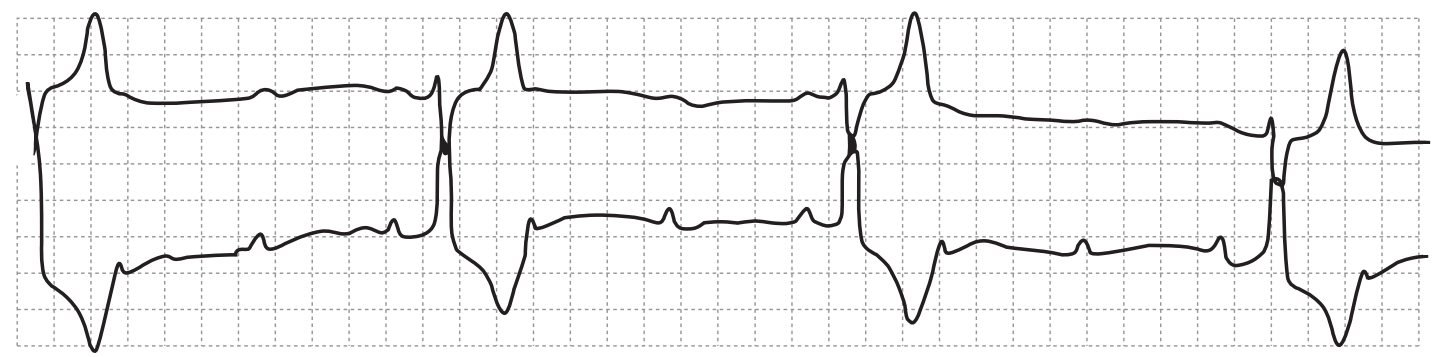

Figure 2. This figure illustrates complete heart block with an atrial rate of 76 beats per minute and an idioventricular pacemaker with a ventricular rate of 26 beats per minute

and this may be the initial presentation in $40 \%$ of patients with cardiac sarcoidosis [19]. Ventricular tachycardia is also one of the most frequent arrhythmia noted in cardiac sarcoidosis, and one study demonstrated a $23 \%$ incidence of ventricular tachycardia (Table I) [20]. Sarcoid granulomas may serve as foci for abnormal automaticity or to disperse both ventricular activation and recovery processes that can cause reentrant tachycardias. The reentrant pathway can result from active granulomatous inflammation but also can be found in association with the healing of cardiac granulomas in the inactive phase of the disease. Atrial arrhythmias are less common than ventricular arrhythmias, occurring in $15-17 \%$ of cases. They are often the result of atrial dilatation or pulmonary involvement rather than the result of atrial granulomas [12].

\section{Congestive heart failure}

Progressive heart failure accounts for $25 \%$ to $75 \%$ of cardiac deaths in patients with cardiac sarcoidosis [21]. Heart failure may be secondary to left- sided cardiac involvement with either systolic or diastolic dysfunction and can occur when there is extensive infiltration of the myocardium by noncaseating granulomas. Differentiating cardiac sarcoidosis from idiopathic dilated cardiomyopathy (IDC) can be difficult. Yazaki et al. retrospectively compared 15 patients having cardiac sarcoidosis and left ventricular systolic dysfunction to 30 patients with IDC [22]. The sarcoidosis group had a significantly higher frequency of complete heart block ( $67 \%$ vs. $0 \%$ ), right bundle branch block $(57 \%$ vs. $17 \%)$, and abnormal left ventricular wall thickness (73\% vs. 17\%).

\section{Pericardial effusion/valvular involvement/ventricular aneurysm}

Pericardial involvement is uncommon even in the presence of extensive myocardial infiltration. It is observed in fewer than $10 \%$ of patients with cardiac sarcoidosis, and these patients usually remain asymptomatic. Small pericardial effusions detected by echocardiography were found in 19\% of patients with sarcoidosis [23, 24]. Direct valvular involvement occurs in fewer than $3 \%$ of patients, but valvular incompetence secondary to papillary muscle dysfunction can be seen in approximately $68 \%$ of patients [25]. Mitral regurgitation may result from papillary muscle dysfunction or dilatation of the left ventricle secondary to diffuse disease.

Ventricular aneurysms occur in $10 \%$ of the patients with sarcoidosis. The anterior and septal wall segments are more commonly affected, and it is unusual that wall motion of only the apical segment is reduced. Extension of ventricular lesions may lead to aneurysm formation. Long-term corticosteroid therapy can heal sarcoid granulomas, replacing myocardium with fibrous tissue, but steroid therapy has been associated with ventricular aneurysms [26, 27]. Nonetheless, corticosteroids should be used if clinically indicated since ventricular aneurysm is common in untreated patients with myocardial sarcoidosis. Myocardial aneurysms may be associated with frequent and complex ventricular arrhythmias. These arrhythmias may resolve after resection of the aneurysm. Impaired arterial perfusion in the vicinity of granulomas may impair the local delivery of antiarrhythmic drugs. Therapy is also complicated by the presence of certain acidic acute phase reactants that bind to drugs with high $\mathrm{pK}$, thus interfering with serum levels.

\section{Pulmonary hypertension and cor pulmonale}

Pulmonary hypertension $(\mathrm{PH})$ is a predictor of poor outcome in sarcoidosis. Shorr et al. reported that the frequency of $\mathrm{PH}$ was as high as $73.8 \%$ in 363 advanced sarcoidosis patients listed for lung transplant [28]. In a study by Tomhiro et al., the frequency of $\mathrm{PH}$ in Japanese sarcoidosis patients who were followed in an outpatient clinic was $5.7 \%$ [29]. There are multiple mechanisms for $\mathrm{PH}$ in sarcoidosis. Pulmonary hypertension can be a consequence of impaired forward flow and result from poor left ventricular function. Pulmonary hypertension may occur in patients with pulmonary sarcoidosis, especially those with hypoxic vasoconstriction, resulting in cor pulmonale. Alveolar hypoxia, irrespective of the cause, not only 
causes vasoconstriction but can result in pulmonary vascular remodeling. At times elevated pulmonary arterial pressures are associated with normal or near normal lung function and arterial blood gases. This may suggest the presence of an intrinsic sarcoid vasculopathy. Encroachment of the pulmonary vasculature by intimal and medial infiltration by noncaseating granuloma and extrinsic compression of pulmonary arteries by enlarged mediastinal lymph nodes can also cause $\mathrm{PH}$ [30]. Pulmonary hypertension has been associated with more severe pulmonary involvement as reflected by chest $\mathrm{X}$ ray and pulmonary function tests. There may be multiple causes of sarcoid-associated $\mathrm{PH}$, and therefore these patients should undergo a complete and careful diagnostic work-up.

\section{Diagnosis}

Only after a thorough clinical evaluation documenting a syndrome consistent with sarcoidosis and biopsy documentation of the presence of noncaseating granulomas can a diagnosis of sarcoidosis be made. All other causes of non-caseating granulomas must be reasonably excluded. The presence of signs, symptoms, or findings suggestive of cardiac involvement warrant additional assessment to confirm presence of cardiac sarcoidosis [2].

\section{Endomyocardial biopsy}

A definite diagnosis of cardiac sarcoidosis can be made by endomyocardial biopsy. The sensitivity of endomyocardial biopsy for non-caseating granulomas is low, usually less than $20 \%$ [31]. It is believed that this low sensitivity is a consequence of patchy myocardial involvement which frequently involves the intraventricular septum and the left ventricle. Despite its low sensitivity, early myocardial biopsy can be considered when the diagnosis of cardiac sarcoidosis is entertained. Endomyocardial

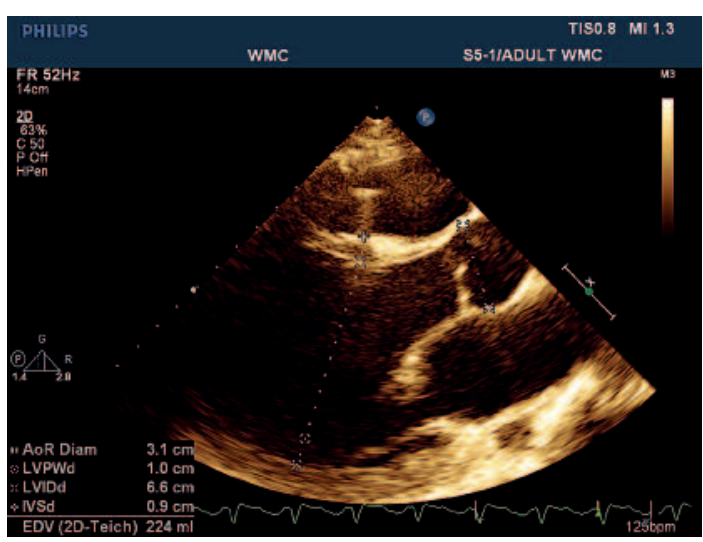

Figure 3. This figure is a 2-dimensional echocardiogram showing a dilated cardiomyopathy. The left ventricular internal dimension at the end of diastole is $6.6 \mathrm{~cm}$ biopsy can help to rule out other causes of cardiac myopathy and may confirm the diagnosis of cardiac sarcoidosis [2]. In the absence of granulomas, it might be difficult to distinguish between cardiac sarcoidosis and idiopathic giant cell myocarditis [32] as both of these diseases have giant cells and are associated with ventricular tachycardia and heart block. The differential diagnosis will include connective tissue diseases like Lyme disease, rheumatoid arthritis, dermatomyositis, cardiac amyloidosis and alcohol related cardiomyopathy, but these can be distinguished based on clinical findings.

\section{Electrocardiographic and Holter monitoring}

The electrocardiogram (ECG) should be part of the routine evaluation of the patient with a diagnosis of sarcoidosis. The electrocardiogram changes are found in as many as $50 \%$ of patients with systemic sarcoidosis. This may occur without clinical evidence of cardiac involvement [33-35]. Although the ECG is an important part of patient evaluation, it is often nondiagnostic or non-specific. In a study by Suzuki et al. 24-h Holter monitoring detected cardiac sarcoidosis with a sensitivity of $67 \%$ and a specificity of $62 \%$ when ventricular ectopic beats numbered more than 100/day [36]. An electrical abnormality even as insignificant as sinus tachycardia should be investigated as it may indicate myocardial infiltration. QT dispersion (QTd), the maximal interlead difference in $\mathrm{QT}$ interval on the surface 12 lead ECG, may be a predictor of sudden cardiac death. A preliminary study of 35 patients with systemic sarcoidosis, the subgroup with cardiac sarcoidosis had a significantly greater QTd than the noncardiac sarcoidosis group and a control group. The incidence of premature ventricular complexes on ECG in a limited followup group was greater in the cardiac sarcoidosis group than in the noncardiac sarcoidosis group [37]. Carvedilol has been reported to significantly reduce QTd [38].

\section{Doppler echocardiography}

Echocardiography is an important tool in the diagnosis and surveillance of patients with cardiac sarcoidosis. In a recent study of 41 patients, Doppler echocardiography was abnormal in $67 \%$ of patients with dilated cardiomyopathy in $32 \%$ of patients (Figure 3), with abnormal left ventricular relaxation in $29 \%$ of patients, and with diffuse or localized dyskinesia or hypokinesia in $26 \%$ of patients [39]. Two-dimensional echocardiographic abnormalities for cardiac sarcoidosis include abnormal septal thickening or thinning, dilatation of the left ventricle (Figure 3), and systolic dysfunction of the left ventricle [25]. Doppler echocardiography may also identify left ventricular diastolic dysfunction which 
can be an early sign of granulomatous involvement of the myocardium [40]. Echocardiography may not detect mild localized myocardial abnormalities which occur in the early stage of cardiac disease. The cycle dependent variation of myocardial integrated backscatter (CV-IB) is another new technique. It can be decreased in the basal septum in patients with cardiac sarcoidosis even in the absence of 2-dimensional echocardiographic abnormalities [41]. Mechanisms may include decreased regional myocardial contraction function, altered myocardial acoustic properties influenced by myocytolysis, and cell infiltration in the myocardium. This may be a useful method to detect early myocardial involvement in patients with sarcoidosis.

\section{Radionuclide studies}

Nuclear medicine imaging is another important tool in the diagnosis of cardiac sarcoidosis. The fibrogranulomatous lesions in the myocardium display segmental areas of decreased uptake in nuclear imaging. Most useful studies are performed with thallium 201 and technetium 99m sestamibi [42]. Improvement or complete resolution with dipyridamole differentiates cardiac sarcoidosis from coronary artery disease in which defects at rest worsen or fail to improve with exercise, dipyridamole, or adenosine. Myocardial perfusion abnormalities in sarcoidosis are reversible after pharmacological dilation which may be due to possible microvascular vasoconstriction in myocardial sarcoidosis. This phenomenon is referred to as "reverse distribution" $[43,44]$.

Thus, in order to assess cardiac sarcoidosis, a baseline resting myocardial scan should be performed in addition to a scan after dipyridamole infusion. Sestamibi single-photon emission computer tomography (SPECT) is more sensitive than thallium in the diagnosis of myocardial sarcoidosis. However this modality is not specific for cardiac sarcoidosis. Gallium is highly sensitive for cardiac sarcoidosis since it accumulates in the inflamed areas and is useful in judging the response of the disease to steroid therapy. Unfortunately, myocardial gallium SPECT images are not sufficiently clear in distinguishing gallium uptake in the myocardium from that in the lung or mediastinum. Therefore, the clinical usefulness of gallium SPECT scanning has not been adequately established [45].

A study by Kasawa et al., showed that the use of dual SPECT scanning with gallium and technetium could be a useful diagnostic imaging technique and improved the diagnostic specificity of gallium SPECT for the diagnosis of cardiac sarcoidosis [46]. Studies have shown that myocardial uptake of gallium may predict responsiveness to steroid therapy [45].

\section{Magnetic resonance imaging}

Sarcoid infiltrates are visible by magnetic resonance imaging $(\mathrm{MRI})$ as a zone of increased intramyocardial signal intensity. These are more pronounced on T2 weighted images because of the edema associated with inflammation and granulomatous lesions. These images can be enhanced on gadolinium diethylene pentaacetic acid enhanced MRI. Focal myocardial thickening is often seen as a result of the edema [47-50].

Increased signal intensity on T2 weighted sequences without myocardial thickening and without gadolinium uptake can also be observed. This feature may be noticed in patients already receiving corticosteroids or on the follow-up MRI after treatment. Delayed enhanced $M R I$ is considered a useful method for the early identification of cardiac sarcoidosis. Delayed hyperenhancement is frequently associated with a reduction of regional wall motion and thallium201 perfusion defects. The area with delayed enhancement represents myocardium that has been replaced by the fibrogranulomatous tissue of sarcoidosis, resulting in decreased wall motion [51].

\section{Positron emission tomography}

Positron emission tomography (PET) can also identify sarcoid cardiac involvement and assess severity. The PET scan may be useful in patients with a pacemaker or cardioverter-defibrillators implanted who are unable to undergo MRI because of the safety concerns related to potential adverse effects on the device arising from the strong magnetic and radiofrequency forces generated by MRI. These include the possibility of erratic and inappropriate device functioning during or after the scan, over-sensing that can cause high rate pacing or thermal damage to the device, and induced voltages on leads that can cause over- and undersensing. Moreover, combined effects can cause component failures, mechanical vibration, and device damage [52].

Yamigishi et al. studied 17 patients with cardiac sarcoidosis using PET [53]. Positron emission tomography imaging was positive in 14 (82\%) of these patients [53]. Only 6 of 17 patients (35\%) showed 201 thallium defects, and only 3 of 17 patients (18\%) revealed abnormal 67 gallium accumulation. In addition, the patients who were treated with steroids showed a decrease in uptake and hence improvement on PET. Positron emission tomography has the potential to be an important tool in the diagnosis and monitoring of patients with cardiac sarcoidosis.

\section{Other studies}

Cardiac catheterization is indicated in patients with sarcoidosis to rule out coronary artery disease 
in patients with chest pain, congestive heart failure, or abnormal imaging. In the presence of normal coronary arteries, the perfusion defects on thallium scanning in a patient with sarcoidosis strongly point toward the existence of cardiac sarcoidosis. However, sarcoid patients with cardiac dysfunction, ECG abnormalities, or thallium-201 imaging defects should be presumed to have cardiac sarcoidosis even when endomyocardial biopsy shows no granulomas.

Cardio-pulmonary exercise studies, including measurement of peak oxygen consumption, can be very helpful in the evaluation for heart failure [54].

\section{Treatment and prognosis}

\section{Immunosuppressive therapy}

Cardiac sarcoidosis is an indication for treatment because of increased risk of sudden death. Treatment is given to reduce inflammation. Corticosteroids, the most common initial therapy, should be started in patients with a definite or strong probability of cardiac sarcoidosis on different imaging studies, even with a negative myocardial biopsy. Chiu et al. showed that long- term steroid use in patients with a left ventricular ejection fraction (LVEF) > 55\% may prevent LV remodeling and altered cardiac function. Steroids most benefited those patients with a LVEF $<54 \%$ who showed significant reduction in LV volumes and LVEF improvement [55]. In patients with a LVEF $<30 \%$, steroid therapy did not improve the LV volume or function. These investigators concluded that in early or middle stage disease, steroid therapy may be protective or therapeutic but may not be as effective in the late stages.

The severity of congestive heart failure is the most powerful prognostic predictor of survival in steroid treated patients with cardiac sarcoidosis. Yazaki et al. reported that the 5-year survival was about $90 \%$ in steroid treated patients with preserved systolic function [21, 22]. There was no significant difference in survival curves for patients treated with a high initial dose vs. a low dose of steroids. It is reasonable to start a steroid dose with prednisone $60-80 \mathrm{mg}$ a day and taper the dose over 6 months to a dose of 10-mg a day. If the disease appears to be improved and dormant, then the steroids can be slowly tapered further. At any sign of recurrence, steroids should be started at the dose of $60 \mathrm{mg} /$ day or higher. Long-term corticosteroid therapy is often required.

Smedema et al. concluded that once symptomatic cardiac sarcoidosis develops in pulmonary sarcoidosis patients, the prognosis becomes very poor. In contrast with asymptomatic cardiac sarcoidosis, the prognosis of asymptomatic cardiac involvement in patients with pulmonary sarcoidosis is better. Therefore, it is appropriate to screen pulmonary sarcoidosis patients with an electrocardiogram and clinical evalution and reserve more specialized cardiac evalution for those with abnormalities [56].

Treatment with methotrexate, azathioprine, or cyclophosphamide can be used as steroid sparing agents and in those whose disease is refractory to high-dose steroids [57, 58]. Tumor necrosis factor is critical in the genesis and maintenance of granulomatous inflammation. Case reports have described cardiac conduction abnormalities which improved after use of infliximab, a tumor necrosis factor alpha inhibitor [59]. There is no consensus on the duration of therapy, and recurrence can occur after cessation of therapy. There are no reported randomized controlled trials describing the superiority of one treatment over another. A commonly used approach would be to start treatment with corticosteroids with addition of immunosuppressive agents in refractory cases. In cases where steroids are contraindicated, use of immunosuppressive agents would be given as initial therapy.

\section{Other therapies}

Ventricular tachycardia is a common complication, and steroids have usually failed to prevent ventricular tachycardia. Antiarrhythmic drug therapy for ventricular tachycardia in patients with cardiac sarcoidosis is associated with a high rate of recurrence or sudden death. Amiodarone use in this group of patients may be limited by the occurrence of pneumonitis and/or pulmonary fibrosis. These drug-induced pulmonary changes are indistinguishable radiographically from pulmonary sarcoidosis and may compromise the patients' respiratory status. $\beta$-Blockers have been shown to increase the incidence of heart block in patients with cardiac sarcoidosis. Given these limitations, implantation of an implantable cardioverter defibrillator should be considered as primary therapy in such patients. The indications for permanent pacing are similar to those in patients without cardiac sarcoidosis. Permanent pacing is effective in the prevention of sudden cardiac death and in advanced atrioventricular block and other bradyarrhythmias.

\section{Cardiac transplantion}

Cardiac transplantation is reserved for end-stage disease unresponsive to medical therapy [60]. Major indications for cardiac transplantation are resistant ventricular tachyarrhythmias and severe intractable heart failure, especially in younger patients. Cardiac sarcoidosis results in progressive fibrosis and scarring leading to ventricular dysfunction. Patients 
with stage $D$ heart failure who are refractory to treatment with angiotensin converting enzyme inhibitors, angiotensin receptor blockers, $\beta$-blockers and diuretics should be considered for cardiac transplantation [61]. Cardiac transplantation can be avoided if corticosteroid treatment is started before the occurrence of severe systolic dysfunction. Sarcoidosis can recur in the transplanted heart and has been documented to recur 24 weeks to 19 months after transplantation. Transmission of sarcoidosis from an allograft donor, who was not known to have sarcoidosis, to its recipient has been reported [62]. An increased dosage of steroids may be required to achieve complete resolution in all recurrences after transplantation.

\section{Acknowledgments}

The authors have no significant financial interest or other relationship with any product manufacturer or provider of services discussed in this article.

\section{References}

1. Benjamin AR, Marcie M, John P, et al. Racial differences in sarcoidosis incidence: a 5-year study in a health maintenance organization. Am J Epidemiol 1997; 145: 234-41.

2. Lee LS, Rose CS, Maier LA. Sarcoidosis N Engl J Med 1997; 336: 1224-34.

3. Bernstein M, Konzelmann FW, Sidlick DM. Boeck's sarcoid: report of a case with visceral involvement. Arch Intern Med 1929; 44: 721-34.

4. Silverman KJ, Hutchins GM, Buckley BH. Cardiac sarcoid: a clinicopathologic study of 84 unselected patients with systemic sarcoidosis. Circulation 1978; 58: 1204-11.

5. Tadamura E, Yamamuro M, Kubo S, et al. Effectiveness of delayed enhanced MRI for identification of cardiac sarcoidosis: comparison with radionuclide imaging. AJR Am J Roentgenol 2005; 185: 110-5.

6. Matsui Y, Iwai K, Tachibana T, et al. Clinicopathological study on fatal myocardial sarcoidosis. Ann N Y Acad Sci 1976; 278: 455-69.

7. Tachibana T, Iwai K, Takemura T. Study on the cause of death in patients with sarcoidosis in Japan [abstract]. XII World Congress on Sarcoidosis, Kyoto, Japan, September 8, 1991.

8. Newman LS, Rose CS, Bresnitz EA, et al. A case control etiologic study of sarcoidosis: environmental and occupational risk factors. Am J Respir Crit Care Med 2004; 170: 1324-30.

9. DuBois RM, Goh N, McGrath D, et al. Is there a role for microorganisms in the pathogenesis of sarcoidosis? J Int Med 2003; 253: 4-17.

10. Tercelj M, Rott T, Rylander R. Antifungal treatment in Sarcoidosis - a pilot intervention trial. Resp Med 2007; 101: 774-8.

11. Doughan AR, Williams BR. Cardiac Sarcoidosis. Heart 2006; 92: 282-8.

12. Roberts WC, McAllister HA, Ferrans VJ. Sarcoidosis of the heart. A clinicopathologic study of 35 necropsy patients (group I) and review of 78 previously described necropsy patients (group II). Am J Med 1977; 63: 86-108.
13. Baughman RP, Teirstein AS, Judson MA, et al. Clinical characteristics of patients in a case control study of sarcoidosis. Am J Respir Crit Care Med 2001; 164: 1885-9.

14. Naruse TK, Matsuzawa Y, Ota M, et al. HLA-DQB1*0601 is primarily associated with the susceptibility to cardiac sarcoidosis. Tissue Antigens 2000; 56: 52-7.

15. Takashige N, Naruse TK, Matsumori A, et al. Genetic polymorphisms at the tumour necrosis factor loci (TNFA and TNFB) in cardiac sarcoidosis. Tissue Antigens 1999; 54: 191-3.

16. Sharma OP. Diagnosis of cardiac sarcoidosis: an imperfect science, a hesitant art. Chest 2003; 123: 18-9.

17. Fleming HA. Sarcoid heart disease. Br Heart J 1974; 36 : 54-68.

18. Yoshida Y, Morimoto S, Hiramitsu S, et al. Incidence of cardiac sarcoidosis in Japanese patients with high-degree atrioventricular block. Am Heart J 1997; 134: 382-6.

19. Sekiguchi $M$, Numao $Y$, Imai $M$, et al. Clinical and histological profile of sarcoidosis of the heart and acute idiopathic mypcarditis. Concepts through a study employing endomycoardial biopsy. I Sarcoidosis. Jpn Circ J 1980; 44: 249-63.

20. Furushima $H$, Chinushi $M$, Sugiura $H$, et al. Ventricular tachyarrhythmia associated with cardiac sarcoidosis: its mechanisms and outcome. Clin Cardiol 2004; 27: 217-22.

21. Yazaki Y, Isobe M, Hiroe $M$, et al. Prognostic determinants of long-term survival in Japanese patients with cardiac sarcoidosis treated with prednisone. Am J Cardiol 2001; 88: 1006-10.

22. Yazaki Y, Isobe M, Hiramitsu S, et al. Comparison of clinical features and prognosis of cardiac sarcoidosis and idiopathic dilated cardiomyopathy. Am J Cardiol 1998; 15: 537-40.

23. Garrett J, O'Neill H, Blake S. Constrictive pericarditis associated with sarcoidosis. Am Heart J 1984; 107: 394.

24. Pesola G, Teirstein AS, Goldman M. Sarcoidosis presenting with pericardial effusion. Sarcoidosis 1987; 4: 42-4.

25. Lewin RF, Mor R, Spitzer S, et al. Echocardiographic evaluation of patients with systemic sarcoidosis. Am Heart J 1985; 110: 116-22.

26. Lull RJ, Dunn BE, Gregoratos G, et al. Ventricular aneurysm due to cardiac sarcoidosis with surgical cure of refractory ventricular tachycardia. Am J Cardiol 1972; 30: 282-7.

27. Jain A, Starek PJ, Delany DL. Ventricular tachycardia and ventricular aneurysm due to unrecognized sarcoidosis. Clin Cardiol 1990; 13: 738-40.

28. Shorr AF, Davies DB, Nathan SD. Predicting mortality in patients with sarcoidosis awaiting lung transplantation. Chest 2003; 124: 922-8.

29. Handa T, Uozato H, Higa R, et al. Incidence of pulmonary hypertension and its clinical relevance in patients with sarcoidosis. Chest 2006; 129: 1246-52.

30. Preston I, Klinger JR, Landzberg MJ, et al. Vasoresponsiveness of sarcoidosis-associated pulmonary hypertension. Chest 2001; 120: 866-72.

31. Uemura A, Morimoto S, Hiramitsu S, et al. Histologic diagnostic rate of cardiac sarcoidosis: evaluation of endomyocardial biopsies Am Heart J 1999; 138: 299-302.

32. Okura Y, Dec GW, Hare JM, et al. A clinical and histopathologic comparison of cardiac sarcoidosis and idiopathic giant cell myocarditis. Am J Cardiol 2003; 41: 322-8.

33. Stein E, Jacler I, Stimmel B, et al. Asymptomatic electrocardiographic alterations in sarcoidosis. Am Heart J 1973; 86: 474-7.

34. Schuster EH, Conrad G, Morris F, et al. Systemic sarcoidosis and electrocardiographic conduction abnormalities. Chest 1980; 78: 601-4. 
35. Thunell M, Bjerle P, Stjemberg N. ECG abnormalities in patients with sarcoidosis. Acta Med Scand 1963; 213: 115-8.

36. Suzuki T, Tsugiyasu K, Kubata S, et al. Holter monitoring as a noninvasive indicator of cardiac involvement in sarcoidosis. Chest 1994; 106: 1021-4.

37. Uyarel H, Uslu N, Okmen E, et al. QT dispersion in sarcoidosis. Chest 2005; 128: 2619-25.

38. Tatli E, Aktoz M, Gul C, et al. Does carvedilol therapy reduce $\mathrm{QT}$ dispersion in patients with heart failure? Arch Med Sci 2008; 4: 404-8.

39. Chapelon-Abric C, de Zuttere D, Duhaut $P$, et al. Cardiac sarcoidosis: a retrospective study of 41 cases. Medicine 2004; 83: 315-34.

40. Skold CM, Larsen FF, Rasmussen E, et al. Determination of cardiac involvement in sarcoidosis by magnetic resonance imaging and Doppler echocardiography. J Intern Med 2002; 252: 465-71.

41. Hyodo E, Hozumi T, Takemoto Y, et al. Early detection of cardiac involvement in patients with sarcoidosis by a noninvasive method with ultrasonic tissue characterization. Heart 2004; 90: 1275-80.

42. Haywood LJ, Sharma OP, Siegel ME. Detection of myocardial sarcoidosis by thallium-201 imaging. J Natl Med Assoc 1983; 63: 478-82.

43. Fields CL, Ossorio MA, Roy TM, et al. Thallium 201 scintigraphy in the diagnosis the management of myocardial sarcoidosis. South Med J 1990; 83: 339-42.

44. Tellier P, Paycha F, Antony I, et al. Reversibility by dipyridamole of thallium-201 myocardial defects in patients with sarcoidosis. Am J Med 1988; 85: 189-93.

45. Okayama K, Kurata C, Tawarahara K, et al. Diagnostic and prognostic value of myocardial scintigraphy with thallium 201 and gallium 67 in cardiac sarcoidosis. Chest 1995; 107: 330-4.

46. Nakazawa A, Ikeda K, Ito Y, et al. Usefulness of dual 67Ga and $99 \mathrm{mTc}$-sestamibi single- photon-emission CT scanning in the diagnosis of cardiac sarcoidosis. Chest 2004; 26: 1372-6.

47. Vignaux O. Cardiac sarcoidosis: spectrum of MRI features AJR Am J Roentgenol 2005; 184: 249-54.

48. Vignaux O, Dhote $R$, Duboc $D$, et al. Detection of myocardial involvement in patients with sarcoidosis applying T2-weighted, contrast-enhanced and cine magnetic resonance imaging: initial results of a prospective study. J Comput Assist Tomogr 2002; 26: 762-7.

49. Serra JJ, Monte GU, Mello ES, et al. Cardiac sarcoidosis evaluated by delayed-enhanced magnetic resonance imaging. Circulation 2003; 107: 188-9.

50. Matsuki M, Matsuo M. MR findings of myocardial sarcoidosis. Clin Radiol 2000; 55: 323-5.

51. Tadamura E, Yamamuro M, Kubo S, et al. Effectiveness of delayed enhanced MRI for identification of cardiac sarcoidosis: comparison with radionuclide imaging. AJR Am J Roentgenol 2005; 185: 110-5.

52. Kalin R, Stanton MS. Current clinical issues for MR scanning of pacemaker and defibrillator patients. Pacing Clin Electrophysiol 2005; 28: 328-8.

53. Yamagishi $H$, Shirai $N$, Takagi $M$, et al. Identification of cardiac sarcoidosis with (13)N-NH(3)/(18)F-FDG PET. J Nucl Med 2003; 44: 1030-6.

54. Williams SG, Barker D, Goldspink DF, et al. A reappraisal of concepts in heart failure: central role of cardiac power reserve. Arch Med Sci 2005; 1: 65-74.

55. Chiu CZ, Nakatani S, Zhang G, et al. Prevention of left ventricular remodeling by long-term corticosteroid therapy in patients with cardiac sarcoidosis. Am J Cardiol 2005 95: 143-6.

56. Smedema JP, Snoep G, van Kroonenburgh MP, et al. Cardiac involvement in patients with pulmonary sarcoidosis assessed at two university medical centers in the Netherlands. Chest 2005; 128: 30-5.

57. Lower EE, Baughman RP. The use of low dose metho trexate in refractory sarcoidosis. Am J Med Sci 1990; 299: 153-7.

58. Baughman RP, Winget DB, Lower EE. Methotrexate is steroid sparing in acute sarcoidosis: results of a double blind, randomized trial. Sarcoidosis Vasc Diffuse Lung Dis 2000; 17: 60-6.

59. Baughman RP, Lower EE. Infliximab for refractory sarcoidosis. Sarcoidosis Vasc Diffuse Lung Dis 2001; 18: 70-4

60. Valantine HA, Tazelaar HD, Macoviak J, et al. Cardiac sarcoidosis: response to steroids and transplantation. Heart Transplant 1987; 6: 244-50.

61. Statement on sarcoidosis: joint statement of the American Thoracic Society (ATS), the European Respiratory Society (ERS) and the World Association of Sarcoidosis and Other Granulomatous Disorder (WASOG) adopted by The ATS Board of Directors and the ERS Executive Committee, February 1999. Am J Respir Crit Care Med 1999; 160: 736-55.

62. Burke WM, Keogh A, Maloney PJ, et al. Transmission of sarcoidosis via cardiac transplantation. Lancet 1990; 336: 1579. 